\title{
Equilibrium helium film in the thick film limit
}

\author{
J. Klier, F. Schletterer, and P. Leiderer \\ Department of Physics, University of Konstanz, Konstanz D-78457, Germany \\ V. Shikin \\ Institute of Solid State Physics of the Russian Academy of Sciences \\ Moscow District, Chernogolovka 142432, Russia \\ E-mail: shikin@issp.ac.ru
}

\begin{abstract}
For the thickness of a liquid or solid quantum film, like liquid helium or solid hydrogen, there exist still open questions about how the film thickness develops in certain limits. One of these is the thick film limit, i.e., the crossover from the thick film to bulk. We have performed measurements in this range using the surface plasmon resonance technique and an evaporated Ag film deposited on glass as substrate. The thickness of the adsorbed helium film is varied by changing the distance $h$ of the bulk reservoir to the surface of the substrate. In the limiting case, when $h \rightarrow 0$, the film thickness approaches about $100 \mathrm{~nm}$ following the van der Waals law in the retarded regime. The film thickness and its dependence on $h$ is precisely determined and theoretically modeled. The equilibrium film thickness behaviour is discussed in detail. The agreement between theory and experiment is very good.
\end{abstract}

PACS: 67.70.+n, 68.15.+e, 68.43.-h, 68.55.-a

\section{Introduction}

The thickness of a liquid film grown under complete wetting conditions on a horizontal substrate is an important parameter for many areas of condensed matter physics, especially for surface science studies. This film thickness, under thermodynamical equilibrium conditions (i.e., in coexistence with its saturated vapour pressure), is very dependent on the distance of the bulk liquid level to the surface of the substrate, see Fig. 1. The existing description of thick adsorbed films [1,2] generally deals with van der Waals forces. In the case when retardation plays a role the dependence of the film thickness, $d$, is

$$
d \propto h^{-1 / 4} .
$$

However, the singularity in definition (1), when $h \rightarrow 0$, is not physical, i.e., $d$ would go to infinity although the bulk level is just at the height of the substrate (see Fig. 2). This shows that this limiting case has to be described more accurately, see below.

There are some alternative interpretations to the van der Waals dependence of $d(h)$. One of them can be formulated as the meniscus problem. It is well known that the vertical substrate walls can lift some amount of the liquid above the bulk level due to the competition between Laplace force and gravitational force [3], a scenario known as suspended films. The same mechanism could, in principle, be responsible for the creation of a macroscopically thick liquid film when $h \rightarrow 0$ without the participation of van der Waals forces (see Figs. 3,4).

In addition to the theoretical discussion we have performed precise measurements of the thickness of a

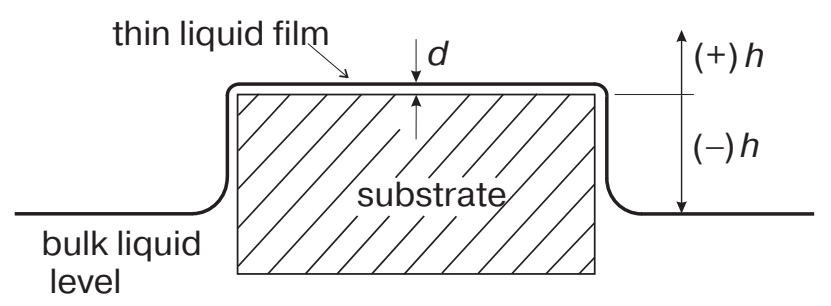

Fig. 1. The thickness of a thin liquid film, $d$, completely wetting a horizontal substrate and being in coexistence with its saturated vapour pressure, i.e., in the presence of bulk liquid. The distance between the substrate and the bulk liquid level is $h$. 
liquid helium film on a silver substrate using the surface plasmon technique [4]. The experimental dependence of $d(h)$, for $h \rightarrow 0$, follows the corrected van der Waals scenario.

\section{Theoretical description}

1. First we will consider the general van der Waals scenario for the behaviour of $d(h)$. If the substrate, on which a helium film is adsorbed, is perfect (i.e. ideally flat), then the definition of the thickness of this film, $d$, in the van der Waals approximation is given by

$$
\left(\frac{k_{w}}{d}\right)^{4}=d-h .
$$

Here $k_{w}$ is the van der Waals constant including retardation. For the definition of $h$ see Fig. 1. Typical values of $k_{\tau}$ are of the order $10^{-6} \mathrm{~cm}^{5 / 4}$ [5].

Under the conditions $h>0$, the solution of Eq. (2) with respect to $d$ is possible provided $h \leq d$. So we get

$$
d \simeq h+\left(\frac{k_{w}}{h}\right)^{4}, \text { for } h>>\left(\frac{k_{w}}{h}\right)^{4} .
$$

At the special point $h=0$, i.e., when the bulk level is at the height of the substrate, we have

$$
\left(\frac{k_{w}}{d_{0}}\right)^{4}=d_{0} \text {, or } d_{0}=k_{w}{ }^{4 / 5} .
$$

For the bulk helium level below the substrate, i.e., when $h<0$, we get

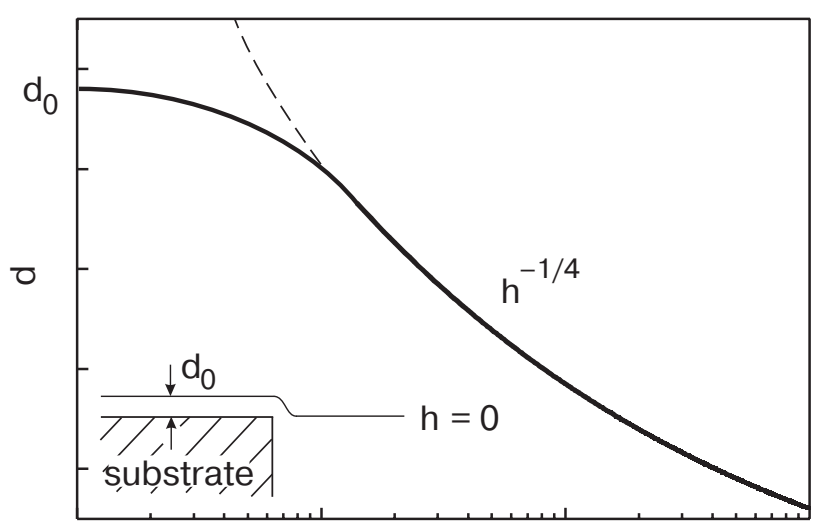

$\log h$

Fig. 2. The dependence of $d(h)$ in the van der Waals approximation (qualitatively), considering Eqs. (4) and (5) in a semi-log scale. The dashed line indicates the behaviour of $d(h)$ if it is only described by Eq. (1). The inset illustrates the limiting case when $h=0$, and so the thickness of the adsorbed film is $d_{0}$.

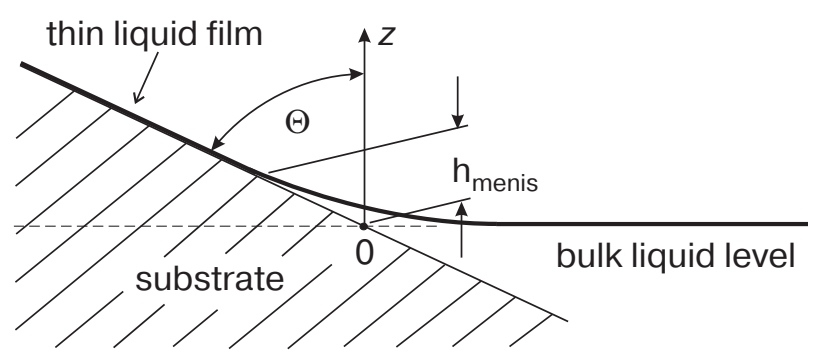

Fig. 3. A tilted substrate, with tilt angle $\Theta$ which is partially immersed in bulk liquid. The upper part of the substrate, i.e., for $h>0$, is covered by a thin liquid film. However, just above the value $h=0$ the liquid film is lifted by the meniscus forming between the substrate wall and the bulk liquid level. The height $h_{\text {menis }}$ up to which the film is governed by the meniscus, is described by Eq. (6).

$$
\left(\frac{k_{w}}{d}\right)^{4}=-h, \text { for } h<0 .
$$

The predicted behaviour of $d(h)$ in the van der Waals approximation is presented in Fig. 2.

2. The lift of the thin liquid film by the bulk meniscus is estimated using the geometry shown in Fig. 3.

The calculations, like in Ref. 3, show that

$$
h_{\text {menis }}=a(1-\sin \Theta)^{1 / 2} \text { with } a^{2}=\frac{2 \sigma_{l v}}{\rho g} .
$$

Here $\rho$ is the liquid density, $g$ the acceleration due to gravity, $\sigma_{l v}$ the liquid helium surface tension, and $a$ the capillary length. It becomes evident that for the limiting case $\Theta \rightarrow \pi / 2$ then $h_{\text {menis }}<<a$. Therefore, a lift of the liquid by the meniscus in case of an ideal wetted horizontal substrate is not effective.

3. However, if the solid substrate is not flat (which is usually the case), then there is another channel for a

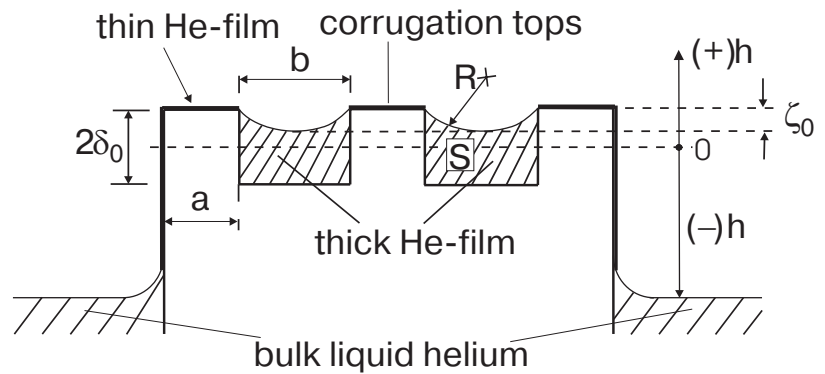

Fig. 4. A schematic sketch of a corrugated surface where, due to the meniscus effect, a suspended thick liquid helium film can be formed. The symbols are explained in the text. 


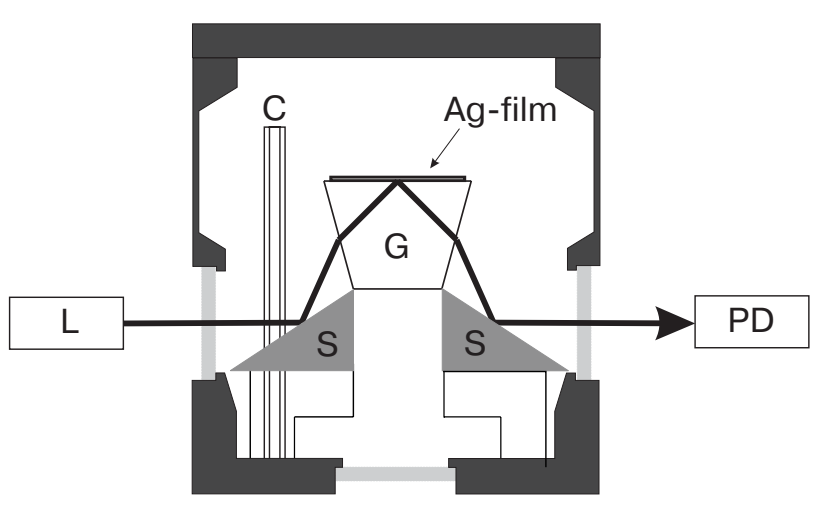

Fig. 5. The scketch of the experimental cell. An incoming light beam from a monochromatic source $L$ is reflected from a mirror $S$ towards the face of a glass prism $G$. From there the reflected light is detected by a sensitive photodiode $P$. The adsorbed helium is measured on top of a thin $40 \mathrm{~nm}$ thick Ag film evaporated onto the glass prism. The bulk helium level (not shown) is measured via a cylindrical capacitor $C$ standing vertically inside the cell. Temperature equilibrium is checked both by a thermometer mounted onto the prism surface and via the vapour pressure measured with a high resolution pressure gauge outside the cryostat.

$d(h)$ dependence. This arises also from the meniscus effect [3]. Now we will formulate this dependence for a corrugated perturbation of the surface of the solid substrate, see Fig. 4. In this case we have

$$
<d(h)>=\frac{S(h)}{a+b}+\frac{a}{a+b} \tilde{d}(h),
$$

where

$$
S=2 \delta_{0} b-\frac{1}{2}\left[l R-b\left(R-\zeta_{0}\right)\right]
$$

and

$$
l=\sqrt{b^{2}+\frac{16}{3} \zeta_{0}}, \quad \zeta_{0}=R-\sqrt{R^{2}-\frac{b^{2}}{4}}, \quad R(h)=\frac{2 \sigma_{l v}}{\rho g h} .
$$

Here $\tilde{d}(h)$ represents the van der Waals contribution, from Eq. (5), in $<d>$.

Under the condition $R(h)>>b$ the value $S(h)$, Eq. (8), is not sensitive to $h$, and so the dependence $d(h)$ can be presented as

$$
\begin{gathered}
<d(h)>\simeq d_{\text {cor }}+\tilde{d}(h) \frac{a}{a+b}, \\
\text { with } d_{\text {cor }} \simeq \frac{2 \delta_{0} b}{a+b},
\end{gathered}
$$

with the asymptotic behaviour of $\tilde{d}(h)$ as

$$
\begin{aligned}
& {\left[\frac{k_{w}}{\tilde{d}(0)}\right]^{4} \simeq \delta_{0}+\tilde{d}(0) \quad \text { for } h \rightarrow 0,} \\
& {\left[\frac{k_{w}}{\tilde{d}(h)}\right]^{4} \simeq h \text { for } h>>\tilde{d}(0)+\delta_{0} .}
\end{aligned}
$$

One can see that the meniscus effect $S(h)$ in $<d(h)>$ can be dominant if $\delta_{0}>d_{0}$, where $d_{0}$ is from Eq. (4). It can also be sensitive to $h$ if the Laplace radius $R(h)$ is comparable with the characteristic of the corrugation $b$. In the opposite limit, Eq. (10), the presence of roughness of the solid substrate (more precisely - solid corrugation) leads to a shift of the film thickness in the dependence $<d(h)>$.

\section{Experimental verification}

In order to check above predictions for the limiting case when $h \rightarrow 0$ we have performed preliminary measurements of the thickness of a growing ${ }^{4} \mathrm{He}$ film. As experimental technique we used a surface plasmon (SP) resonance setup which allows for a resolution of the helium film thickness of about $1 \AA$, see Ref. 4. The surface plasmons are excited, using a monochromatic light source, at the interface of a thin quench-condensed Ag film, deposited on a glass prism, and the adsorbed helium film, see Fig. 5. This light beam is reflected at the prism and the reflected light is measured with a very sensitive photodiode (for a more detailed description of such a setup see Ref. 6). Under resonance conditions, i.e., when the angle of incidence corresponds to the surface plasmon resonance angle, little or no light is detected. Keeping this resonance conditions via a feedback loop the thickness of the adsorbed helium film can be measured.

The thickness of the helium film is determined as function of the bulk helium level. This bulk level is changed in small steps by slowly condensing in helium gas from a known volume. It turns out that the relaxation times to achieve a stable bulk level is of the order of hours. The experiment is performed at $1.4 \mathrm{~K}$, i.e., when the helium is superfluid. The height of the bulk level is measured with a cylindrical capacitor, see Fig. 5, which gives a resolution of about $50 \mu \mathrm{m}$. This height measurement is cross-checked by the total volume of gas added to the cell and a precise check of the cell volume and the inside geometry after the experiment.

In Fig. 6 we show the growth of the helium film starting from a bulk level of $(-) h=0.5 \mathrm{~cm}$ below the surface of the substrate. At this point the thickness of the helium film $d$ is about $73 \mathrm{~nm}$. As the bulk level is raised the film thickness grows showing a $h^{-1 / 4}$ dependence as described by Eq. (10)-(12). Within these 


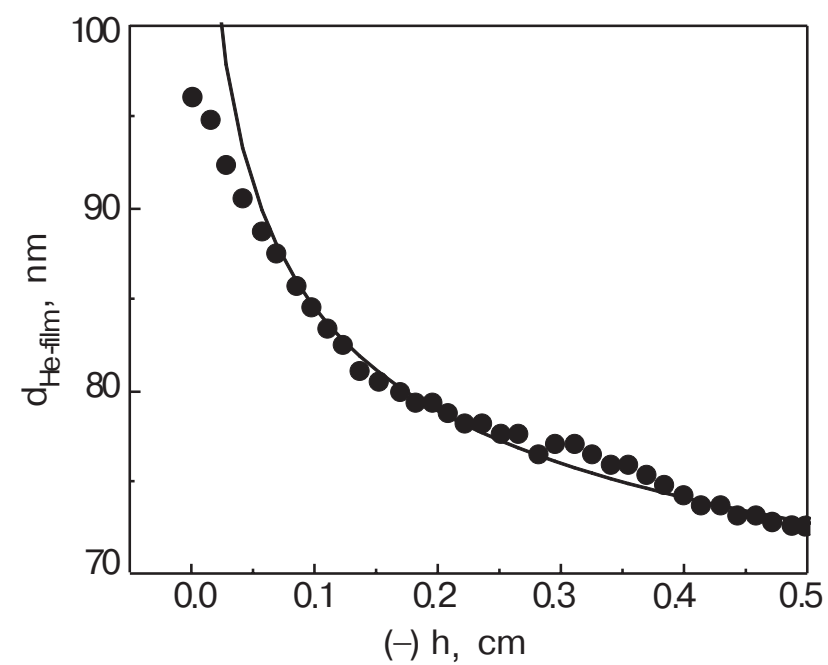

Fig. 6. The thickness of the adsorbed helium film, $d$, as function of the varying bulk helium level, $h$. Here $h$ is already very small and so the dependence of $d$ follows the $h^{-1 / 4}$ law (solid line) for $h \geq 0.06 \mathrm{~cm}$, see text. For smaller values of $h$ i.e. $<0.1 \mathrm{~cm}$ the film thickness deviates from this law.

results we can fit our experimental data. When fitting the data in the interval $0.1 \mathrm{~cm}<(-) h<0.3 \mathrm{~cm}$, using Eq. (10), the agreement is quite good, see Fig. 7 , and the parameter $d_{\text {cor }}$ can be obtained. It turns out that $d_{\text {cor }} \approx 49 \mathrm{~nm}$ and so (if we assume that $a \approx b$ ) we get $\delta_{0} \simeq d_{\text {cor }}=49 \mathrm{~nm}$.

We interpret this observation due to the fact that the surface must be very rough, but without Gaussian peaks, and so there is bulk condensation between some roughness peaks which adds to the measured film thickness. That our surface was indeed quite rough was seen in the measured SP resonance curves which showed a large resonance width, much wider than for an ideal smooth Ag-film. However, once the adsorbed helium film is thicker than the height of the roughness peaks its influence is screened and so a further growth in film thickness should show the ideal behaviour, seen in the data for $h \rightarrow 0$. The influence of substrate roughness in the film thickness of adsorbed films will be investigated and presented elsewhere.

When $h$ drops below $0.1 \mathrm{~cm}$ the measured data deviate from the above law, see Fig. 6. Eventually the film thickness levels off at around $100 \mathrm{~nm}$, see Fig. 7 . Using Eq. (11) and $k_{w} \approx 6.4 \cdot 10^{-7} \mathrm{~cm}^{5 / 4}$ one gets for $\tilde{d}(0) \approx 102 \mathrm{~nm}$. From Eq. (10) we get $d(0) \simeq d_{\text {cor }}+$ $+0.5 \tilde{d}(0)=100 \mathrm{~nm}$, which corresponds well to the experimental value for $h \rightarrow 0$.

\section{Conclusions}

We have investigated the growing of an adsorbed liquid film $d$ on a substrate as function of the distance

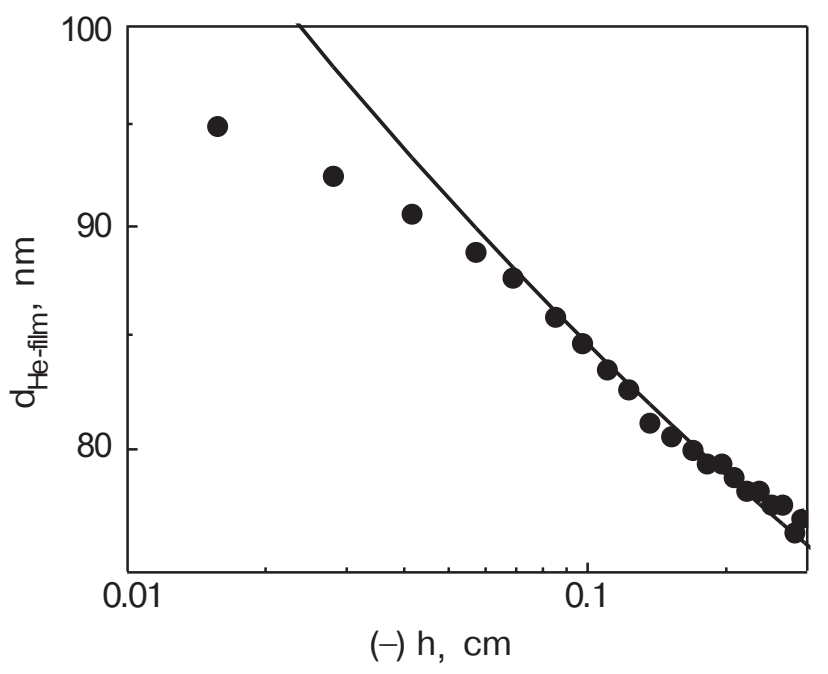

Fig. 7. The same data points of $d$ plotted against $h$ as in Fig. 6, however now on a semi-log plot. The deviation from the expected $h^{-1 / 4}$ behaviour (solid line) for small values of $h$ is clearly seen. The thickness of the helium film approaches a final value of around $100 \mathrm{~nm}$. This is the predicted behaviour shown in Fig. 2, and described by Eqs. (4) and (5).

between the bulk liquid level and the surface of the substrate $h$ which is above the bulk level. The calculations of the thickness of such a film are given for the thick film limit, i.e., when $h \rightarrow 0$ and under retardation conditions, on ideal smooth surfaces. These predictions are confirmed by measurements of a liquid helium film adsorbed to a silver surface. Both the thickness of the helium film and the change in the bulk helium level are measured with high resolution in the range of $70 \mathrm{~nm}<d<100 \mathrm{~nm}$ for $0.5 \mathrm{~cm}>(-) h>0$. Experiments to study the cross-over from the retarded to the non-retarded regime are currently running. The influence of substrate roughness on adsorbed quantum films is also under investigation, both theoretically and experimentally.

This work was supported by the DFG-Schwerpunkt 'Wetting and Structure Formation at Interfaces' under Kl 1186/1.

1. H.B.G. Casimir and D. Polder, Phys. Rev. 73, 360 (1948).

2. L.W. Bruch, M.W. Cole, and E. Zaremba, Physical Adsorption: Forces and Phenomena, Clarendon Press, Oxford (1997); and references therein.

3. L.D. Landau and E.M. Lifshitz, Hydrodynamics, Akademie Verlag, Berlin (1991).

4. D. Reinelt, J. Klier, and P. Leiderer, J. Low Temp. Phys. 113, 805 (1998).

5. E.Y. Andrei, Phys. Rev. Lett. 52, 1449 (1984).

6. V. Iov, J. Klier, and P. Leiderer, J. Low Temp. Phys. 126, 367 (2002). 